\title{
The Study of Neuropsychological Functions in Myopes, Following an Intervention of Trataka Yoga Kriya
}

\author{
Uday K. Dixit ${ }^{1}$, Sushma S. ${ }^{2}$ \\ ${ }^{1}$ Third Year MBBS Student, ${ }^{2}$ Assistant Professor, Department of Physiology, \\ Bangalore Medical College and Research Institute, Fort, K.R. Road, Bangalore-560 002, Karnataka
}

\begin{abstract}
Aim of Study: To evaluate the effects of Trataka yoga on neuropsychological functions in myopic subjects.

Methodology: Out of 36 myopes recruited for the study, 25 subjects were considered for statistical analysis. Digit Span Test and Complex Figure test were measured before and after an intervention of Trataka yoga kriya for 3 weeks.

Results: In the present study, post Trataka yoga kriya intervention, Complex figure test results showed an increase in mean scores from 31.32 to 34.20 and Digit span test results showed an increase in mean scores from 7.32 to 7.96 , which was found to be statistically significant.

Conclusion: Our study concluded that Trataka yoga has a significant effect on neuropsychological functions in myopes Further regular Trataka yoga kriya practice can be used as an inexpensive, simple and nonpharmacological means as a protective factor against cognitive deterioration.
\end{abstract}

Keywords: Myopes, Trataka yoga, Neuropsychological functions.

\section{Introduction}

Myopia is one of the most prevalent disorders of the eye and is associated with comorbidities with an estimated $22.9 \%$ of the world population, being affected. ${ }^{1}$ An additional $2.7 \%$ of people are estimated to have high myopia. ${ }^{2}$ The economic impact of uncorrected refractive error is estimated to be a loss of \$202 billion of global gross domestic product. In 2010, just over $28 \%$ of the world's population were affected by Myopia. This is predicted to rise to $34 \%$ by 2020 and nearly $50 \%$ by $2050 .^{3}$

\section{Corresponding Author:}

\section{Dr. Sushma S.}

Assistant Professor, Department of Physiology, Bangalore Medical College and Research Institute, Fort, K.R. Road, Bengaluru-560002

e-mail: suvina76@gmail.com

Phone: 9916915194
Cognitive functions also termed Neuro Psychological functions are the particular psycho mental processes within a person's psyche that are present regardless of common circumstance. ${ }^{4}$ Older myopics were almost twice as likely to have cognitive dysfunction when compared with individuals with emmetropia ${ }^{5}$ due to amyloid and acetyl choline deficiency.

Trataka Yoga Kriya is a form of Yoga believed to improve eyesight and strengthen ocular muscles.Many studies have proved that yoga is effective to improve various cognitive functions such as remote memory, mental balance, attention and concentration, attention span, processing speed, attention alternation ability, and recognition tests in the healthy young subjects. ${ }^{6}$

However, a very few studies have been able to relate the cognitive abilities involving visuospatial memory skills and refractive error in myopes. The purpose of our study is to evaluate this possible association in myopics aged 18 to 25 years following a non-pharmacological intervention of Trataka yoga kriya. 
Digit span scores and cognitive functions improved immediately after the practice of Trataka in the healthy elderly subjects. ${ }^{7}$ Another study by Raghavendra et al has seen positive changes in the Neuropsychological functions of subjects post intervention of Trataka Yoga $\mathrm{Kriya}^{8}$. Anna $\mathrm{H}$ et al have studied the use of EEG oscillations to predict cognitive status in a cohort of healthy males with varying cognitive ability. ${ }^{9}$

Aims and Objectives: To study the effects of Trataka yoga kriya on the Neuropsychological functions in myopes.

\section{Material and Method}

Study design: Pre and Post intervention study.

Study period: August 2019 and October 2019

Place of study: Dept. of Physiology, BMCRI.

Study group: The study was conducted on 36 subjects prediagnosed with myopia.

Sample size: 36 subjects prediagnosed with myopia

\section{Inclusion Criteria:}

1. Subjects in the age group of 18 and 25 years.

2. Subjects pre-diagnosed with Myopia.

\section{Exclusion Criteria:}

1. History of any ocular surgery

2. History of any ocular pathology

3. Subjects with colour blindness.

4. Subjects with history of seizures

5. History of neurological/psychiatric and other systemic diseases

6. Subjects who had received yoga training in the previous 3 months.

Methodology: Written informed consent was obtained from the recruited subjects after explaining the procedure in detail. The subjects were selected based on inclusion and exclusion criteria and their base line data was recorded.

Assessment of Neuropsychological Functions: A cognitive function involving visuospatial skills was tested by Complex Figure Test and working memory for sustained attention was tested by digit span test.
Complex Figure Test or Rey-Osterrieth Complex figure test: The Rey-Osterrieth Complex Figure Test (ROCF) is a widely used neuropsychological test for the evaluation of visuospatial constructional ability and visual memory. The ROCF consists of three test conditions: Copy, Immediate Recall and Delayed Recall. At the first step, subjects were given the ROCF stimulus card, and then asked to draw the same figure. Subsequently, they were instructed to draw what they remembered. Then, after a delay of $30 \mathrm{~min}$, they were required to draw the same figure once again. The results were scored according to location, accuracy and organization. Each condition of the ROCF takes $10 \mathrm{~min}$ to complete and the overall time of completion is about 30 min..$^{10}$

Digit Span Test: The Digit Span (DS) is a subtest in Wechsler Adult Intelligence Scale-third edition (WAISIII) and has been standardized for use in an Indian population. ${ }^{11}$

A digit-span task is used to measure working memory's number storage capacity. Subjects were shown a sequence of numerical digits and were tasked to recall the sequence correctly, with increasingly longer sequences being tested in each trial. The longest number of sequential digits that can accurately be remembered is the digit span of the subject. Digit-span tasks are the most commonly used test for memory span, partially because performance on a digit-span task cannot be affected by factors such as semantics, frequency of appearance in daily life, complexity, etc. ${ }^{12}$

Trataka Yoga Kriya And Related Eye Exercises: After measuring the cognitive ability an intervention of Trataka yoga kriya ${ }^{13}$ and a set of eye exercises was be given for a period of 3 weeks. It includes gazing at the candle flame with focused attention followed by defocusing, breathing and chanting. Each session was for 30 min duration and was conducted on everyday basis.

\section{The preparatory Eye Exercises include}

- Left and Right movements

- Up and Down movements

- Clockwise and anticlockwise Circular movements

- Simple Palming

- Simple and Intermittent Pressure application

- Palming with Brahmari 
The form of Trataka which was practised is the Jyothi Trataka. The subjects were asked to sit 3 feet from a candle flame in a dimly lit room. They were asked to gaze and focus effortlessly and later intensively on the outer part and later the inner parts of the flame with intermittent chanting. In between the exercises simple palming was done to relax the eyes.

\section{Statistical analysis:}

Data was entered in Microsoft Excel and spss version 24.0 was used for statistical analysis. Data was analysed by descriptive statistics such as mean, median, standard deviation and interquartile range, percentage, tables and graphs wherever necessary. Student ' $t$ ' test was used to determine significant difference in neuropsychological functions between pre and post intervention.

\section{Observation and Results}

Of the total 36 subjects, 25 subjects (with a mean power of left and right eyes being $2.29 \pm 1.92$ and
$2.52 \pm 1.97$ respectively both male and female in the ratio of 17:8 with a mean age of 20 years)(Table 1)completed the intervention and were considered for the study. All 25 subjects had successfully completed 3 weeks of yoga intervention and were asked to continue the practice till the readings required for the study were completed.

Pre-intervention tests showed a Complex figure test result mean of 31.31 with SD of 2.60 and digit span test results had a mean of 7.31 with a SD of 0.79.(Fig 1) (Table 1)

Post-intervention Complex figure test results showed a mean of 34.20 with SD of 2.04 and Digit span test results showed a mean of 7.96 with SD of 0.79.(Fig 2) (Table 1)

Paired sample tests show a mean increase of $2.88 \pm 2.74$ and $0.64 \pm 0.86$ between pre intervention and post intervention for Complex Figure Test and Digit Span Test respectively both of which are significant. (Table 2).

Table 1: Pre and Post intervention Comparison

\begin{tabular}{|l|c|c|c|}
\hline Parameter & Pre intervention & Post intervention & Sig (2 tailed) \\
\hline Male : Female ratio* & $17: 8$ & & \\
\hline Power (left)(D)* & $2.29 \pm 1.92$ & & \\
\hline Power (Right)(D)* & $2.52 \pm 1.97$ & & \\
\hline Complex figure test (max-36) & $31.32 \pm 2.66$ & $34.20 \pm 2.04$ & 0.000 \\
\hline Digit span test & $7.32 \pm 0.80$ & $7.96 \pm 0.79$ & 0.001 \\
\hline
\end{tabular}

*Parameters were not considered for post interventional analysis

Table 2: Paired Sample Tests

\begin{tabular}{|l|c|c|}
\hline \multirow{2}{*}{ Test (Pre-Post)* } & \multicolumn{2}{|c|}{ Paired Differences } \\
\cline { 2 - 3 } & Mean & Sig (2-tailed) \\
\hline Complex figure test & $-2.88 \pm 2.74$ & 0.000 \\
\hline Digit Span Test & $-0.64 \pm 0.86$ & 0.001 \\
\hline
\end{tabular}

*The difference between Pre intervention and Post intervention test results is calculated and significance is determined. 


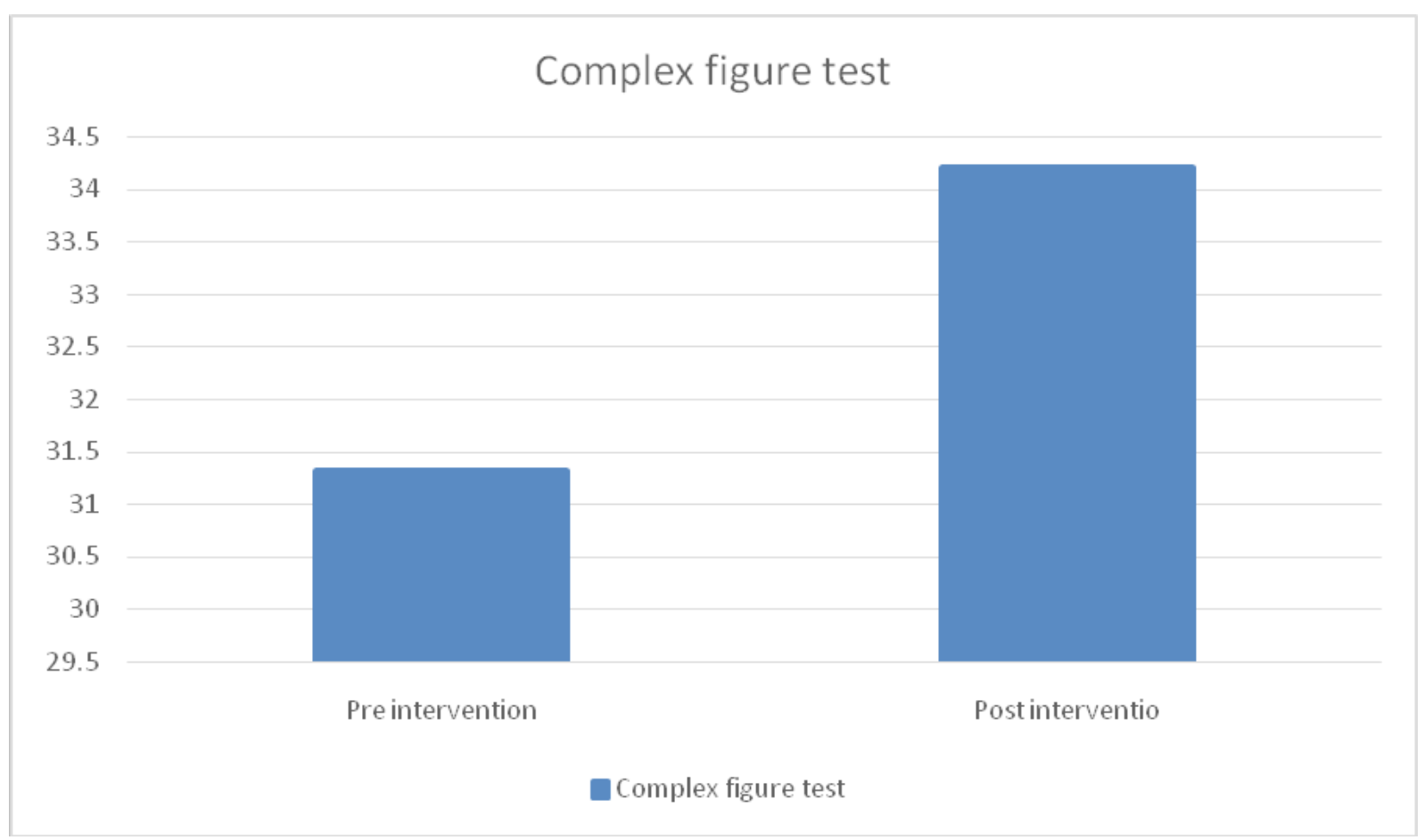

Fig. 1: Mean Complex Figure Test scores

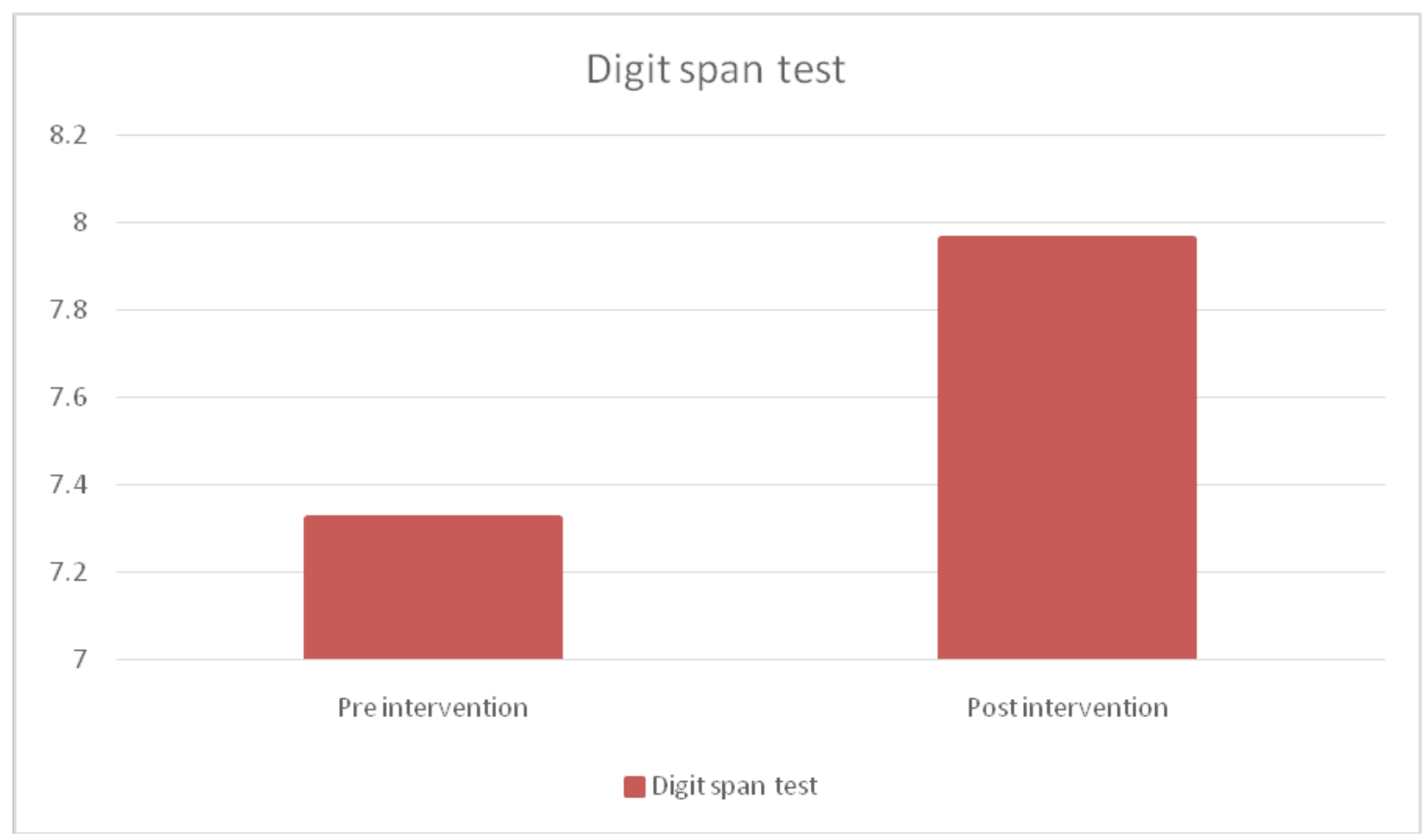

Fig. 2: Mean Digit Span Test scores

\section{Discussion}

The results of the present study demonstrated that Trataka intervention significantly correlated with a better cognitive function score (reflected by improved working memory for sustained and focused attention, visio- spatial skills for visual memory for executive functions). The results are consistent with the results of earlier studies conducted on elderly subjects who showed that, at the end of 3 month follow-up, yoga group improved in semantic memory, short-term primary memory, and 
short-term working memory. ${ }^{14}$ Meditation processes are linked to gamma-aminobutyric acid(GABA) ergic cortical inhibition which improved the cognitive performance and enhanced emotional regulation. ${ }^{15}$

Further, meditation may potentially strengthen neuronal circuits and enhance cognitive reserve capacity. Brain regions associated with attention, interception, and sensory processing are thicker in meditation practitioners including the Prefrontal Cortex and right anterior insula. ${ }^{16}$

Recording the refractive error using Autorefractometers could have been proved to show valid and repeatable measures, which we could not precisely measure due to technical restraints. The use of standardized protocols for obtaining cognitive evaluations were followed by single researcher, to increase the test reliability. Though the Trataka intervention period was only for 3 weeks, still we could show significant improvement in cognitive functioning post intervention. This claim however needs to be tested in larger samples with long term intervention, which could have depicted significant changes. Further studies can be conducted to test the effect of Trataka on different neurological test batteries.

By observing the obtained results, the limitations would be lack of a larger sample size, short interventional protocol duration, the Trataka being tiresome for some of the participants to perform, as they were asked to perform the eye exercises in proper repetition.

\section{Conclusion}

The present study concludes a statistically significant improvement in cognitive function scores following Trataka intervention in myopes. Further regular Trataka yoga kriya practice can be used as an inexpensive, simple and non-pharmacological means as a protective factor against cognitive deterioration. Trataka can be practiced independently by the participant to achieve desired results. For researchers, this study could provide a substantial base for conducting future trials to test the efficacy of Trataka to enhance cognition in myopes.

Acknowledgement: My heartfelt thanks to all the participants for kindly cooperating during the study. I am grateful to Bangalore Medical College and Research Institute for providing the opportunity and support to the budding doctors in the area of research.

\section{Conflict of Interest: Nil}

Source of Funding: Self

Ethical Clearance: Taken

\section{References}

1. Chua J, Wong TY. Myopia-the silent epidemic that should not be ignored. JAMA Ophthalmol. 2016; 134(12):1363-1364.

2. Bourne RR, Stevens GA, White RA, et al. Causes of vision loss worldwide, 1990-2010: A systematic analysis. Lancet Glob Health. 2013; 1(6):339-349.

3. Fricke TR, Holden BA, Wilson DA, et al. Global cost of correcting vision impairment from uncorrected refractive error. Bull World Health Organ. 2012; 90(10):728-738.

4. Jung CG. Psychological Types, Collected Works, Vol 6. New Jersey: Princeton University Press. 1971; 11: 602.

5. Ong SY, Ikram MK, Haaland BA, et al. Myopia and cognitive dysfunction: the Singapore Malay Eye study. Invest Ophthalmol Vis Sci 2013; 54:799-803.

6. Prakash R, Dubey I, Abhishek P, Gupta SK, et al. Long-term Vihangam Yoga meditation and scores on tests of attention. Percept Mot Skills. 2010; 110:1139-48.

7. Shubhada T, Aarti J, Nagarathna R. Int J Yoga. Effect of Trataka on cognitive functions in the elderly.2014; 7(2): 96-103.

8. Raghavendra BR, Singh P. Immediate effect of yogic visual concentration on cognitive performance. $\mathrm{J}$ Tradit Complement Med 2015; 6(1):34-6.

9. Anna Horwitz, Dyhr Thomson, Weigand I, et al. Visual steady state in relation to age and cognitive function. PLoS One. 2017; 12(2):28.

10. Shin MS, Park SY, Park SR et al. Clinical and empirical applications of the Rey-Osterrieth Complex Figure Test. Nature Protocols. 2006; 1(2):892-9.

11. Wechsler DA. Wechsler Adult Intelligence Scale. 3rd ed. San Antonio: The Psychological Corporation; 1997.

12. Jones, Gary, Macken, Bill. Questioning shortterm memory and its measurement: Why digit span measures long-term associative learning. Cognition. 2015; 144: 1-13. 
13. Pancham Sinh, Hatha Yoga Pradipika. First edn. New Delhi: Munshiram Manoharlal Publishers Pvt. Ltd; 2007; 2: 18.

14. Lutz A, Slagter HA, Rawlings NB, et al. Mental training enhances attentional stability: Neural and behavioral evidence. J Neurosci. 2009; 29:1341827.
15. Guglietti CL, Daskalakis ZJ, Radhu $\mathrm{N}$ et al. Meditation-related increases in GABAB modulated cortical inhibition. Brain Stimul. 2013; 6:397-402.

16. Lazar SW, Kerr CE, Wasserman RH et al. Meditation experience is associated with increased cortical thickness. Neuroreport. 2005; 16:1893-7. 